\title{
HACIA UNA CARACTERIZACIÓN DEL ENGAÑO EN EL CONTEXTO DE LA TEORÍA DE DINÁMICA DE TROPAS
}

\begin{abstract}
Mario Barahona Quesada*
\section{RESUMEN}

En este artículo, se intenta avanzar hacia a una primera caracterización del engaño desde la perspectiva de las ciencias cognoscitivas, específicamente en el contexto de la Teoría de Dinámica de Tropas (TDT). Después de aclarar brevemente algunos de los principios básicos en que se fundamenta la TDT, se ofrece un panorama general sobre la presencia y las distintas manifestaciones del engaño observables en la naturaleza. Específicamente, nuestra propuesta consiste en la identificación de seis niveles o momentos de engaño organizados en torno de los mecanismos cognitivos y las predisposiciones instintivas que posibilitan este fenómeno.

Palabras clave: engaño, mentira, procesos cognitivos, ciencia cognitiva, teoría de dinámica de tropa
\end{abstract}

\begin{abstract}
In this paper, we intend to move towards a first characterization of deception from the perspective of cognitive science, specifically in the context of the Theory of Troop Dynamics (TTD). After clarifying briefly some of the basic principles underlying the TTD, we provide a general overview of deception in nature and the various ways in which it manifests itself. Specifically, our proposal consists in the identification of six levels or moments of deception organized around the cognitive mechanisms and instinctive predispositions that make this phenomenon possible.
\end{abstract}

Key Words: deception, lie, cognitive processes, cognitive science, theory of troop dynamics.

\section{Introducción}

Si bien el engaño ha sido un tema abundantemente estudiado en el contexto de muy diversas disciplinas, por ejemplo, la filosofía, el estudio del comportamiento animal, la sociobiología, la psicología evolucionista, la psicología social y la neurociencia, a la luz de avances recientes en ciencia cognitiva, la gran mayoría de estos esfuerzos parecen aún fragmentarios y carentes de una visión conjunta que permita comprender el fenómeno en toda su dimensión. En particular, se trabaja exclusivamente sobre ciertos aspectos del engaño, privilegiando algunas de sus variantes en detrimento de otras, y persiste una brecha significativa entre la forma de abordarlo en nuestra especie y el modo de hacerlo en las demás ${ }^{1}$.

Consideramos que la Teoría de Dinámica de Tropas (TDT, en adelante) — tal y como intentaremos describirlaenelapartado siguienteofrece un marco conceptual apropiado a partir del cual podemos aproximarnos al engaño

* Investigador del Instituto de Investigaciones en Ciencia Cognitiva (IICC) y del Programa de Investigación en Fundamentos de la Educación a Distancia (PROIFED), Universidad Estatal a Distancia. Recepción: 08/05/12. Aceptación: 24/09/12. 
tomando en cuenta tanto la diversidad de sus manifestaciones a lo largo de la naturaleza como los mecanismos cognitivos y representacionales involucrados en su producción. Siendo así, en el presente trabajo, pretendemos esbozar los rudimentos de una primera caracterización del engaño fundamentada en principios de la TDT. Específicamente, nuestra propuesta se organiza en torno de seis niveles o momentos, los cuales representan de manera gradual cómo el aumento en la complejidad de las capacidades cognitivas induce formas cada vez más sofisticadas de engaño. Nótese que el plantear grados o niveles de engaño constituye, en nuestro caso, simplemente un recurso metodológico, pues ciertamente nos acercamos al engaño entendiéndolo como una estrategia adaptativa de naturaleza esencialmente continua a través de las distintas formas de interacción entre los seres vivos.

\section{La Teoría de Dinámica de Tropas ${ }^{2}$}

La TDT es un posicionamiento teórico que intenta dar cuenta de los procesos cognitivos superiores de Homo sapiens sapiens a partir de la consideración de la historia evolutiva, las predisposiciones instintivas y los formantes esenciales de la estructuración social de la especie. A diferencia de otras propuestas en lo que actualmente se conoce como psicología evolucionista - área de las ciencias cognoscitivas que se aproxima a la mente humana a través de los procesos filogenéticos que han modelado su arquitectura (Adenzato y Ardito, 1999: 7) - , la TDT gravita precisamente sobre la noción fundamental de tropa como estructura organizacional básica de las especies del orden de los primates y, por consiguiente, de la nuestra, y a cuya particular complejidad obedece, en gran medida, el desarrollo sofisticado de nuestro aparato cognitivo superior. Así como el grado de complejidad de cualquier tropa de monos (simiformes) supera al de prácticamente cualquier otro tipo de organización (por ejemplo, el cardumen, la bandada o la manada) entre las especies sociales, así una tropa de seres humanos es indudablemente más compleja que la de cualquiera de sus parientes evolutivos más cercanos (Arce, 2008: 111). Al permitir albergar una mayor cantidad de individuos, la ventaja adaptativa que confiere una estructura social compleja (por ejemplo, en contextos de depredación o cooperación) trae, entonces, como consecuencia la aparición de un entorno flexible y constantemente cambiante producto de la interacción entre los miembros del grupo, lo cual ejerce una presión selectiva muy poderosa para que progresivamente se lleguen a desarrollar capacidades cognitivas de orden superior (Arce, 2008: 121).

En contraste con otras formas de organización social (por ejemplo, de tipo piramidal líder alfa, ya sea de naturaleza patriarcal como se presenta entre los gorilas, ya matriarcal como se da entre las hienas manchadas, o de tipo piramidal pareja alfa, como aparece entre los lobos) en donde las relaciones se caracterizan por ser fuerte o rígidamente jerárquicas, una tropa humana exhibe jerarquías débiles, y su arquitectura organizacional se denomina toroidal grupo alfa (Arce, 2011: 75-76). Esto quiere decir, por una parte, que las posiciones de liderazgo son indiferentes en términos de sexo, y pueden ser reasumidas por miembros que, en determinado momento, las hayan perdido (Arce, 2011: 75); y por otra, que las más importantes relaciones de subordinación se dan primariamente entre grupos, no entre individuos particulares (Arce, 2008: 111). Por ello, los mecanismos mediante los cuales se articulan naturalmente las posiciones dentro de una tropa dependen más de estrategias de negociación y manipulación que de la fuerza bruta o la intimidación (Arce, 2008: 111).

Una estructura toroidal grupo alfa induce, entonces, una serie de tensiones intrínsecas que requieren de resolución constante por parte de los integrantes de nuestra especie, y a las que sólo es posible hacer frente apelando a recursos simbólicos, esto es, lingüísticamente mediados (Arce, 2011: 77). Entre estas tensiones, quizá las más significativas sean “...individuo/colectividad, locus individual/ locus colectivo, incompatibilidad entre algoritmos de apareamiento (...), competencia/ 
cooperación [y] cambio/estabilidad" (Arce, 2011: 77). A grandes rasgos, estas tensiones se refieren respectivamente a las siguientes contradicciones: (a) al mismo tiempo que un ser humano intenta afirmar su individualidad diferenciándose de los demás, depende para todo propósito práctico del grupo al que pertenece y al cual debe adecuarse necesariamente; (b) mientras que la individualidad se define en relación con los otros (posee un locus colectivo), la colectividad sólo existe materializada en los individuos particulares que la conforman (su locus es individual); (c) en tanto que el algoritmo de apareamiento entre los machos consiste en impregnar la mayor cantidad posible de hembras, el algoritmo entre las hembras consiste en escoger para sí el mejor macho; (d) si bien al interior de una tropa los instintos predominantes son los de cooperación, existen también instintos competitivos promovidos principalmente por las relaciones entre tropas distintas; y (e) aunque la adaptabilidad y la flexibilidad ante el cambio son esenciales para la supervivencia, ninguna adaptación exitosa se da sin estabilidad y continuidad temporal (Arce, 2011: 77-78). Claramente, hallar un equilibrio en el seno de este conjunto de tensiones resulta imposible en ausencia de capacidades cognitivas de alto nivel que sustenten el desarrollo de mecanismos de negociación, manipulación, postergación de la gratificación y manejo de la ambigüedad, entre otros, los cuales operan tanto en el contexto de la tropa como a lo interno del propio individuo.

Consecuentemente, en el marco de la TDT se postula la existencia de tres arquitecturas cognicionales en torno de las cuales se organiza nuestro aparato cognitivo superior, a saber: modelamiento lingüístico (ML), modelamiento de la mente (MdM) y modelamiento de interrelaciones e interacciones de tropa (MiiT) (Arce, 2010: 67-68, 2011: 79). Cada una de estas arquitecturas, entendidas como conjuntos de patrones agentivos y representacionales, a su vez, ofrece acomodo o acota respectivamente a uno de los siguientes mecanismos de base instintiva o genética: el lenguaje natural (L), la teoría de la mente (TdM) y la inteligencia maquiavélica (IMq) (Arce, 2011: 79). Si bien no fue hasta el advenimiento de la especie Homo sapiens sapiens (hace unos 200 mil años), y específicamente a la aparición de ML, que las arquitecturas de MdM y MiiT se configuraron propiamente como tales, podemos retrotraer la datación de su sustrato filogenético varios millones de años atrás. Así, por ejemplo, los rudimentos de una $\mathrm{TdM}$ primate deben de haber estado presentes hace más de 12 millones de años, antes de la separación entre la línea de la que descienden los orangutanes y la que dio origen al resto de los grandes simios (incluida nuestra especie), del mismo modo en que una IMq incipiente debió de haber aparecido no hace menos de 30 millones de años, en fecha anterior a la separación entre los monos del nuevo mundo y los monos del viejo mundo (Byrne, 2003: 57-58).

A diferencia de los mecanismos de base instintiva o genética, los cuales operan sobre insumos provenientes de la experiencia inmediata, las arquitecturas cognicionales ofrecen la posibilidad de generar modelos del mundo a pequeña, mediana o gran escala, sin la necesidad de verificación directa y con la ventaja de ser modificables tanto en tiempo real como fuera de línea (Arce, 2011: 81). La presencia de estas arquitecturas permite, a su vez, que nuestra especie se desenvuelva simultáneamente en contextos o universos de representación cualitativamente distintos: el universo de la interioridad (UR1), el universo de la interacción con los otros seres humanos (UR2) y el universo de lo que transcurre, hasta cierto punto, independiente de nuestra existencia (UR3) (Arce, 2010: 65-66). De este modo, ML posibilita la modelación de UR1, UR2 y UR3, MdM hace lo propio respecto de UR1 y UR2, y MiiT actúa solamente sobre UR2; lo cual pone en evidencia la preponderancia cognitiva que adquiere la resolución de los problemas y tensiones connaturales al medio social sobre la de los universos interior y físico (Arce, 2011: 79).

Como se verá más adelante, el aparato teórico provisto por la TDT permite articular de manera coherente muchos de los insumos sobre el engaño que han sido generados en el 
marco de distintas disciplinas, así como poner en perspectiva las diferentes manifestaciones de este fenómeno, los mecanismos cognitivos y las predisposiciones instintivas que lo posibilitan y las presiones adaptativas y sociales que hacen de su presencia prácticamente una constante a lo largo de la naturaleza. Si bien la TDT se ocupa en primera instancia del estudio de los procesos cognitivos superiores de nuestra especie, al situar a Homo sapiens sapiens en el contexto de sustrato biológico y evolutivo, hace posible elaborar proyecciones respecto de la cognición en el resto de los primates y, por contraste, en muchas otras especies.

\section{Niveles de engaño ${ }^{3}$}

Sin lugar a duda, la frecuencia y dispersión con que es posible encontrar evidencia de engaño a lo largo del mundo natural revela una selección altamente favorable para este tipo de estrategia adaptativa en los procesos evolutivos de un sinnúmero de especies. Si consideramos, entonces, el engaño como un continuum observable en la naturaleza, cuyo grado de sofisticación aumenta en función de la complejidad de los procesos cognitivos involucrados en su producción; podemos identificar sus manifestaciones más básicas en el extenso repertorio de disposiciones fenotípicas que permiten a ciertos organismos evitar ser detectados o reconocidos (Stevens y Merilaita, 2009: 423) como tales por sus depredadores, competidores, presas u otros seres vivos con los que mantienen relaciones bióticas particulares, lo cual favorece su supervivencia o les facilita la obtención de recursos que, en condiciones distintas, no les serían accesibles o solo difícilmente. Con esto nos referimos, por ejemplo, a las funciones de camuflaje de ciertas especies o a la habilidad de tantas otras para asemejarse (mimetismo) a organismos más dotados o menos amenazantes según corresponda (como es el caso de la serpiente rey o falsa coral, cuyos anillos remiten a los de la mortífera coral). Si bien, como se observa, este tipo de mecanismos se encuentra al margen de lo que podríamos considerar como procesamiento cognitivo o actividad intencional, su carácter primario (esto es, situar a otro en un contexto determinado que otorga ventajas adaptativas mediante la omisión de señales específicas) se mantiene invariante aún en las formas más elaboradas de engaño.

Un segundo momento dentro de este continuum podría estar representado por las respuestas genéticamente determinadas o los comportamientos ritualizados de muchas especies ante situaciones amenazantes, de desventaja competitiva o de caza, por medio de los cuales aumentan sus probabilidades de éxito creando condiciones que, de algún modo, confunden a los otros organismos directamente involucrados. A menudo, este tipo de despliegues incluyen estrategias como las descritas en la categoría anterior (es decir, camuflaje o mimetismo); sin embargo, se distinguen de ellas por presentarse, no como particularidades morfológicas inalterables de la especie, sino como reacciones instintivas a ciertos estímulos relevantes provenientes del exterior (particularmente, las acciones de otros organismos), lo cual implica claramente una coordinación entre percepción y actividad (Mitchell y Thompson, 1986: 24). Siguiendo a Robert Mitchell, en términos computacionales, esto podría describirse como un programa "abierto" de la forma "si $q$ entonces $p$ ", donde $q$ corresponde al estímulo y $p$ a la acción del agente (Mitchell y Thompson, 1986: 29). Algunos ejemplos de ello pueden apreciarse en ciertas aves del orden de las caradriformes o la familia de las colúmbidas, las cuales, al detectar la presencia de un depredador terrestre, abandonan sus nidos y se posan lejos sobre el suelo fingiendo estar heridas para llamar la atención del depredador y alejarlo así de la ubicación de su progenie (McFarland y Bösser, 1993: 188). Asimismo, los crustáceos del orden de los estomatópodos, cuando se encuentran en el período de alta vulnerabilidad que sucede a la muda de su exoesqueleto, suelen proteger sus cuevas de intrusos conespecíficos, exhibiendo un nivel de agresividad que, en condiciones normales, solamente caracteriza a los miembros más fuertes de su especie, de forma que aparentan 
ser capaces de causar daño a sus competidores en un momento en el que claramente no lo son (Hauser, 1997: 119). En este mismo orden de cosas, es posible situar la estrategia de "hacerse los muertos", observable en muchos animales, o la expulsión de tinta de ciertas especies de la clase de los cefalópodos, como los calamares o los pulpos.

En tercer lugar, podríamos referirnos a la habilidad de los miembros de ciertas especies (principalmente entre las que cuentan con algún grado de sociabilidad) para modificar deliberadamente aspectos de su comportamiento habitual o estándar con fines oportunistas, sobre la base de un reconocimiento de la influencia de sus actos sobre la conducta de otros individuos, lo cual presupone capacidades de aprendizaje y memoria particulares (Mitchell, 1993: 69). De acuerdo con Mitchell, este tipo de mecanismo podría representarse formalmente como: "efectuar cualquier acción $p$ dado que $p$ ha resultado en una consecuencia deseada $q$ en el pasado" (Mitchell y Thompson, 1986: 25). A manera de ejemplo, no son poco frecuentes las anécdotas sobre perros domésticos (Canis lupus familiaris), en las que se describe cómo alguno de ellos logra despojar a otros de un bien preciado (dígase, alimento o un espacio específico), valiéndose de acciones que usualmente capturan la atención de los demás $\mathrm{y}$, en consecuencia, los hacen descuidar su situación inmediata; como cuando ladran insistentemente, ya sea mirando por una ventana o dirigiéndose rápido hacia una puerta sin ninguna razón aparente. Si bien, a primera vista, este tipo de engaños pueden producir una impresión de intencionalidad, debe tomarse en cuenta que son intencionales, únicamente en el tanto en que de ellos se espera un resultado determinado, mas no así intencionalmente engañosos, pues esto requeriría de un reconocimiento de los estados mentales del otro (Mitchell y Thompson, 1986: 25).

Por otra parte, la investigación en el campo de la primatología y los avances más recientes en torno de la hipótesis de la inteligencia social, han permitido perfilar una dimensión cognitiva entre los simiformes (monos y grandes simios) —así como en algunas otras especies de familias tan diversas como las de los córvidos, los delfines, los elefantes o las hienas-, que los distingue de manera particular del resto de las especies, y consecuentemente, remite a formas más sofisticadas de engaño. De acuerdo con autores como Richard Byrne o Andrew Whiten, quienes han estudiado con suficiente profundidad el engaño entre los primates, el aumento en el tamaño de los grupos de las especies ancestrales del tronco común de los monos y los grandes simios actuales, producto de la depredación intensiva que sufrieron por su transición hacia la vida diurna hace aproximadamente 30 millones de años, ejerció una presión selectiva por un aprendizaje más rápido y efectivo en contextos sociales (Byrne, 2003: 54, 57). Esto, a su vez, resultó en un incremento paulatino del tejido neocortical de sus descendientes, lo cual se tradujo en una serie de capacidades para rastrear redes de relaciones sociales complejas, reconocer parámetros sociales (como estatus o rango), identificar a otros individuos, sus acciones pasadas y su lugar dentro de la estructura social, percatarse de la disposición espacial del grupo y determinar ¿quién puede ver a quién? y ¿quién puede ver qué? en un momento dado (Byrne, 2003: 54, 58; Byrne y Bates, 2007b: 715). Este conjunto de capacidades, al que comúnmente se hace referencia con el término de "inteligencia maquiavélica" (IMq) y que, según la TDT, constituye el sustrato filogenético de nuestra arquitectura de modelamiento de interrelaciones e interacciones de Tropa (MiiT), hizo posible el desarrollo de la manipulación como estrategia para sobrevivir en medios sociales caracterizados por la formación de alianzas y coaliciones (alianzas de alianzas) (Wilson y Keil, 1999: 495), donde hallar un adecuado balance entre cooperación y competencia se torna imperativo (De Waal, 2001: 267).

Algunas anécdotas ilustrativas de la clase de engaño manipulativo que emplean los monos del viejo mundo en sus interacciones cotidianas se desprenden de observaciones de campo como las siguientes. Según refiere Byrne (Byrne y Whiten, 1990: 47), una cría perteneciente a una de las especies de babuinos (Papio ursinus) 
observaba de cerca cómo una hembra adulta cavaba en la tierra para desenterrar quizás algún tubérculo o raíz comestible. Tras mirar a su alrededor advirtiendo que nadie los veía, la cría comenzó a gritar sin ninguna razón aparente. De pronto, la madre apareció y emprendió una persecución en contra de la otra hembra hasta que ambas se perdieron de vista. Finalmente, la cría se acercó al hueco y continuó con la excavación abandonada. Del mismo modo, el primatólogo Hans Kummer (Byrne y Whiten, 1990, p. 33) relata que en una ocasión pudo observar cómo, inusualmente, una hembra de la especie Papio hamadryas, que se encontraba a la vista del macho dominante de su grupo, tardó un aproximado de 20 minutos para modificar milímetro a milímetro su postura corporal hasta estar en condición de acicalar a otro macho de rango inferior situado detrás de una piedra (ciertamente, una acción que el líder no toleraría). A pesar de que la espalda, cabeza y cola de la hembra resultaban visibles desde la perspectiva del líder, sus brazos y su cara, así como el otro macho permanecían ocultos gracias a la piedra. Como se puede apreciar, este tipo de tramas requieren de todos los componentes cognitivos de IMq mencionados anteriormente, es decir, de una capacidad general para representar y manejar ciertas características de un medio social complejo. En el primer caso, la cría debe, al menos, contar con una representación clara de la ubicación espacial de su madre (lo suficientemente cerca para oír sus gritos, pero sin ángulo suficiente para ver si algo le ocurre) y de la relación entre el rango de esta y el de la otra hembra, pues únicamente un individuo de mayor jerarquía podría amenazar y ahuyentar a otro (Byrne y Whiten, 1992: 622). En el segundo ejemplo, además de la representación de los rangos relativos entre los miembros del grupo, la hembra en cuestión debe ser capaz de asumir lo que Byrne y Whiten denominan como toma de perspectiva visual, esto es, computar lo que el otro puede y no puede ver desde la posición en que se encuentra (Byrne, 2003: 55).

En comparación con el resto de los primates simiformes, el comportamiento de los grandes simios (chimpancés, bonobos, gorilas y orangutanes) refleja ciertas características de orden cognitivo - hasta ahora, sólo observadas paralelamente en ciertas especies de delfines y córvidos-, que apuntan hacia una comprensión más profunda del entorno en que se desenvuelven y hacia una mayor capacidad para formar y manipular representaciones mentales. Entre estas particularidades, sobresalen el aprendizaje de destrezas por imitación y la planificación no verbal, la producción de comportamiento innovador y complejo, la posesión de tradiciones locales, la fabricación y empleo de utensilios ${ }^{4} \mathrm{y}$, en especial, el reconocimiento de las intenciones y los estados mentales de los otros (De Waal, 2001: 149, 162-163, 172), lo que filósofos, psicólogos y estudiosos en muchas otras disciplinas han convenido en llamar "teoría de la mente" (TdM), precursor evolutivo, de acuerdo con la TDT, de nuestra arquitectura de modelamiento de la mente (MdM). Mucho se ha discutido sobre las posibles presiones evolutivas que propiciaron esta diferenciación cognitiva entre los grandes simios y los monos propiamente dichos, pues en promedio, los primeros no cuentan con tropas más numerosas ni poseen una proporción mayor de tejido neocortical por unidad de volumen del resto del cerebro que los segundos (De Waal, 2001: 168, 268). Sin embargo, investigaciones recientes (Byrne y Bates, 2007a; Dunbar y Shultz, 2007a, 2007b; Shultz y Dunbar, 2007; Whiten y van Schaik, 2007) han comenzado a extender lo que tradicionalmente se ha entendido por complejidad social, esto es, cardinalidad de grupo, y han logrado validar con bastante éxito otros parámetros al respeto, como: la estructura organizacional de la especie, el grado de compromiso y la estrechez de los vínculos entre los miembros del grupo, y el efecto de retroalimentación positiva que ejerce la cultura sobre la cognición.

Apartándonos un poco de estas cuestiones, resulta evidente que, en materia de engaño, las habilidades mentales de los grandes simios posibilitan formas de manipulación y planificación de estrategias artificiosas altamente sofisticadas, que trascienden los límites de la 
mera perspectiva visual y se inscriben en el ámbito de la perspectiva mental (Byrne, 2003: 55-58). Ciertamente, la posesión de TdM no sólo permite que los individuos engañen utilizando como insumo la representación de las intenciones, temores, conocimientos y deseos de los otros, sino que también anticipen los resultados de sus acciones en esos mismos términos. A modo de ejemplo, Sue SavageRumbaugh narra el siguiente episodio entre dos chimpancés, que claramente captura la esencia de lo anterior:

A lo largo de los años, Austin ha ido perfeccionando una forma de engañar a Sherman, que utiliza sólo por las noches. Sherman siempre ha tenido miedo de la oscuridad y titubea para salir de noche. Austin, quien es más pequeño que Sherman y está subordinado a él, no le teme a la oscuridad y ha aprendido a utilizar el temor de Sherman para su beneficio propio, haciendo ruidos extraños - particularmente, cuando Sherman lo intimida estando dentro. Por medio de los ruidos, Austin logra revertir, en las noches, el orden de dominancia entre ellos. Lo logra, yéndose hacia afuera y haciendo ruidos inusuales (...). Después de hacer los sonidos, Austin corre hacia adentro y mira hacia afuera como si hubiera algo que temer allá. Sherman, entonces, se asusta, corre y abraza a Austin, y lo deja de intimidar (Byrne y Whiten, 1990:88).

Ahora bien, es necesario hacer notar que, con el desarrollo de TdM, aparecen conjuntamente una serie de rasgos cognitivos que son de particular importancia para la supervivencia en agrupaciones sociales de alta complejidad e interdependencia, tales como, la empatía, el autorreconocimiento (De Waal, 2005: 185) y, casi indudablemente, la introspección. Estas características, ya presentes en los grandes simios, serán precisamente las que abrirán el camino para dos de las manifestaciones del engaño que, en nuestra especie, cristalizarán con extraordinaria ubicuidad: el engaño prosocial, es decir, aquel cuyos fines consisten en producir un contexto de mejor convivencia entre los involucrados, y el autoengaño. Una anécdota ejemplificadora que recoge a la vez ambas facetas, puede apreciarse en el siguiente relato de Frans De Waal (2005: 150) acerca de dos chimpancés hembra, Mama y Kuif, que tenían más de tres décadas de ser amigas. En una oportunidad, recuerda este autor, Mama apoyaba a un "candidato político" entre los machos y Kuif a otro; sin embargo, curiosamente, ellas actuaban como si no supieran de la elección de la otra. Durante los enfrentamientos de poder entre los machos, Mama solía dar un largo rodeo para evitar enfrentarse cara a cara con su amiga, quien se encontraba entre las filas rivales. Dada la indiscutible dominancia de Mama y el extremado mal genio con que acostumbraba tratar a las hembras que no le obedecían, su indulgencia hacia Kuif resultaba una excepción asombrosa.

Finalmente, sin obviar el conjunto de presiones ecológicas y cambios anatómicos que sufrieron los homininos después de su separación de los simios de bosque hace unos 5.5 millones de años ${ }^{5}$, la transición progresiva hacia tropas más numerosas, principalmente en el género Homo, y hacia una estructura toroidal grupo alfa, quizás ya presente desde Homo ergaster, indujo una serie de tensiones que hicieron necesario evolucionar capacidades de representación y procesamiento cognitivo cada vez más complejas y precisas, así como estrategias para garantizar la supervivencia y el éxito reproductivo en entornos flexibles y cambiantes (Arce, 2008: 111). Como respuesta a estas necesidades, en nuestros ancestros se fueron refinando poco a poco IMq y TdM dando lugar a MIT y MdM respectivamentey lentamente se desarrolló la arquitectura de modelamiento lingüístico (ML), alcanzando todo ello su forma actual, hasta donde podemos saber, no antes de 200 mil años al presente con la aparición de Homo sapiens sapiens. Una vez que estas arquitecturas estuvieron plenamente disponibles, nuestra especie contó con la facultad no solo para comprender su medio social y reconocer los estados mentales propios y ajenos, sino también para elaborar sobre la base de estos insumos, compartirlos, organizarlos de acuerdo con categorías simbólicas, modular los potenciales emocionales que evocan (Cartín, 2009: 132), y aplicar estas mismas operaciones sobre el mundo físico y material. En otras palabras, nos vimos en posición para modelar 
y manipular libremente los tres universos de representación que constituyen nuestra noción de realidad: el mundo de la tropa (UR2), el mundo interior (UR1) y el mundo de lo que está allá afuera independientemente de nuestra existencia (UR3).

Aunado a esto, a partir de la interacción coarticulada de las tres arquitecturas cognicionales y del sistema altamente redundante que conforman, emergió en nuestra especie una capacidad sin precedentes para proyectar y manipular escenarios virtuales de manera dinámica e interactiva sin atender a restricciones de viabilidad, lo que comúnmente llamamos imaginación (Arce, 2002: 133). La posibilidad de imaginar asentada, en gran parte, en la referencia desplazada que proporciona ML, además de ser fuente inagotable para la expresividad humana y responsable de fenómenos como el arte, la narrativa, la matemática o la geometría, incidió directamente en nuestras formas más básicas de planificación en tanto que amplió los ámbitos de la prospección, de la introspección y, notablemente, de la retrospección, al permitirnos contemplar espacios y futuros inciertos, asumir personalidades ficticias y reconstruir y modificar el pasado, previendo sus efectos sobre el presente y el porvenir. Si bien, como se ha dicho, otras especies cuentan con cierta habilidad para anticipar y planificar (de manera no verbal), hasta donde sabemos, solamente la nuestra es capaz de hacerlo allende los dominios de lo contingente o lo probable.

Naturalmente, estas particularidades de nuestro aparato cognitivo superior conspiran en favor de la gestación de modalidades de engaño proporcionalmente complejas. En primera instancia, de la mano de ML, aparece de manera paradigmática la mentira, es decir, el engaño mediado y realizado lingüísticamente. Sin embargo, surgen también otras muchas formas de engañar asentadas sobre la naturaleza simbólica y categórica de dicha arquitectura, como, por ejemplo, la falsificación de evidencia (es bien sabido, que solamente nuestra especie es capaz de interpretar indicios, como huellas o rastros) o el disfraz. En las palabras de Terrence Deacon (1997):

\begin{abstract}
Debido a que el vínculo referencial entre los símbolos y sus objetos es indirecto, las mismas características que hacen de la referencia simbólica el único medio para representar definitivamente algo tan abstracto y virtual como una promesa o un contrato también abren la puerta a la tergiversación y la falsedad. (p. 403)
\end{abstract}

Nótese que, aún cuando la mentira se materializa precisamente en la dimensión comunicativa de $M L$, el papel de esta arquitectura no se agota allí; pues, en nuestra especie, todo engaño que involucre cierto grado de dilación (esto excluye, por supuesto, a los engaños constituidos por movimientos corporales rápidos o reflejos aprendidos, tales como los de una finta en el combate o el deporte) requiere, al menos, de otros dos componentes primordiales de $M L$ (Cartín, 2009: 195). Primero, la modelación y evaluación detallada de la realidad propia y ajena (Arce, 2002: 134), en especial, la delimitación clara del contexto compartido con los demás y su contexto complemento, lo cual implica la consideración de expectativas, presuposiciones, creencias, conocimientos, valoraciones, normativas, conceptos, categorías y mecanismos disponibles de verificación, entre otras cosas. Luego, la modulación precisa de los estados emocionales, en cuya ausencia el engaño perdería toda su efectividad, sobre todo cuando ocurre de modo presencial.

Lógicamente, ninguna de estas funciones (comunicación, modelación y modulación) tendría sentido de estar restringidas a la esfera estrictamente individual. De hecho, se encuentran en la base misma de nuestra formidable capacidad para cooperar de manera heterotécnica (habilidad de un grupo para asumir un objetivo común y perseguirlo mediante contribuciones distintas y complementarias por parte de cada uno de los miembros) y no meramente simétrica, como lo hacen otras especies de cazadores (verbigracia, las de la familia de los félidos) (Arce, 2005: 99-100). Esto, a su vez, permite comprender por qué, en nuestra especie, a menudo el engaño es más producto de la participación colectiva (dígase conspiración o encubrimiento) que de la actividad de un solo individuo. 
Por otra parte, las mismas tensiones inducidas por nuestra organización toroidal grupo alfa, responsables en gran medida de las características distintivas de la cognición humana y por consiguiente de nuestras capacidades para engañar, manipular y mentir, son también las causantes de la abrumadora frecuencia con que el engaño aparece en nuestras interacciones cotidianas. De acuerdo con estudios relativamente recientes, durante una conversación de 10 minutos entre extraños, el $60 \%$ de las personas miente en promedio 3 veces, con un mínimo de una mentira y un máximo de 12 por cada encuentro (Feldman, Forrest y Happ, 2002: 166 y 170). Asimismo, según las investigaciones de la doctora Bella DePaulo y sus colegas, los estudiantes universitarios mienten en una de cada tres interacciones con otros individuos, mientras que, en entornos comunitarios, la gente lo hace en una de cada cinco (DePaulo, Kashy, Kirkendol, Wyer y Epstein, 1996: 991). A la luz de la TDT, estas cifras no son sino el reflejo fehaciente de la constante contradicción en que vive sumida nuestra especie. Si recordamos algunas de las tensiones a las que antes nos referimos, por ejemplo, la que gira en torno de la oposición individualidad/colectividad, resulta obvio que hallar un adecuado balance entre estos términos no constituye una tarea para nada trivial, $\mathrm{y}$, aunque en el mejor de los casos pueda ser llevada a cabo con éxito mediante un ejercicio sostenido de autoafirmación, el engaño siempre persiste como la fuente de solución más viable y expedita. Cuando en nuestras relaciones ordinarias con los demás lo que prima es el interés colectivo, el engaño suele manifestarse en su función prosocial, como cuando le decimos a la abuela lo magnífica que ha quedado su sopa mientras la apuramos con abundantes cantidades de bebida para disipar su sabor. Esta clase de engaño se encuentra incluso codificada en muchas de nuestras formas estándar de cortesía, parabienes, halagos y cosas por el estilo. Por el contrario, en los momentos en que el interés individual es el que se sobrepone, ya sea o no en perjuicio de los demás, aparece el engaño egoísta, el cual se extiende desde las versiones modificadas de nuestras calidades personales o acciones pasadas para impresionar a alguien más hasta los distintos tipos de fraude y las calumnias destinadas a deteriorar la reputación de un tercero o a socavar una alianza. Del mismo modo, cuando las tensiones en cuestión representan un problema en el seno de la interioridad, a menudo se recurre al autoengaño como mecanismo para manipular los propios estados mentales e impedir que emerjan a la conciencia los aspectos de la personalidad que producen contrariedad ${ }^{6}$.

De forma paralela, nuestras propensiones simbólica (la necesidad de hallar sentido y sistema en todo lo que hacemos) y categórica (la tendencia a imponer nuestra estructura cognitiva sobre el mundo) nos obligan a llenar los vacíos de nuestra existencia con cuanto creamos suficiente para mitigar la angustiosa incertidumbre connatural a ellas (Arce, 2002, pp. 165-166). Ciertamente, esta particular condición de nuestra especie, constituye un caldo de cultivo sumamente rico para que el engaño prolifere allí donde la realidad se torna desconcertante. Si bien, en términos generales, la eficacia del engaño es proporcional a su grado de adecuación con criterios de viabilidad (p. 134), nuestra incomparable habilidad para desatender los mecanismos de verificación con que estamos dotados (la razón, la referencia de los demás y el insumo de los sentidos) en función de una cierta estabilidad, la confianza natural que depositamos en los otros individuos, en especial, cuando forman parte de la mayoría (de no haber sido así jamás hubiésemos sobrevivido) (Arce, 2004, p. 53) y la fuerza con que nuestra capacidad para imaginar se proyecta sobre la vida misma nos convierten en una especie proclive a creer prácticamente en cualquier cosa. Prueba de ello son los tantos y tantos mitos, supersticiones, falacias pseudocientíficas e ideologías que han desfilado delante de los ojos de la humanidad con estatus de verdades incontrovertibles. Esto pone en evidencia que la búsqueda de la verdad por sí misma no es un imperativo entre los seres humanos, antes bien preferimos una ficción que nos dé seguridad que asumir la duda como una constante. 


\section{Consideraciones finales}

Dado este panorama general, quisiéramos concluir destacando algunos puntos específicos. Como se ha podido observar, nuestra propuesta de caracterización considera seis niveles de engaño articulados en torno de los mecanismos cognitivos y los procesos adaptativos que lo posibilitan, a saber: la selección natural, la coordinación entre percepción y acción, el aprendizaje y la memoria, el rastreo de relaciones sociales complejas promovido por IMq, el reconocimiento de los estados mentales propios y ajenos producto de TdM y la modelación lingüística encarnada en la arquitectura de ML. Naturalmente, cada uno de estos niveles hereda las características de los niveles inferiores, de modo que, por ejemplo, las formas de engaño observables entre los simiformes suponen de antemano disposiciones morfogenéticas, predisposiciones comportamentales y habilidades mnemónicas específicas. No obstante, también parece plausible afirmar que, una vez que se cuenta con capacidades de procesamiento cognitivo de más alto nivel, estas se emplean a fondo para llevar a cabo incluso las funciones más primarias. Sabemos bien que en nuestra especie manifestaciones tan básicas del engaño como lo son el camuflaje o el mimetismo adquieren una nueva dimensión a partir del empleo de recursos simbólicos y tecnológicos. Finalmente, resulta evidente que muchos de los aspectos aquí tratados requerirán de aclaración y elaboración posterior; sin embargo, creemos haber logrado ofrecer un primer esbozo de caracterización del engaño desde la perspectiva de la TDT.

\section{Notas}

1. En otro trabajo se analizan con el debido detenimiento algunas de estas propuestas y se señalan las limitaciones que, desde nuestra perspectiva, experimentan a la hora de aproximarse al fenómeno del engaño.

2. En este apartado, se recogen solamente algunos de los aspectos esenciales contemplados en la Teoría de Dinámica de Tropas (TDT); para un panorama detallado sobre este paradigma explicativo, véase Arce (2011).
3. Los tres primeros niveles incluidos en la clasificación que sigue se deben en gran medida al trabajo de Mitchell y Thompson (1986) y Mitchell (1993); sin embargo, a diferencia de estos autores consideramos todas las formas de camuflaje como engaño propiamente dicho.

4. Para una distinción entre el concepto de utensilios y el de herramientas, véase Arce (2005, pp. 95-114).

5. Para un panorama pormenorizado sobre la evolución de nuestra especie, véase D’Alton (2006).

6. En este sentido, nos alejamos de la interpretación de autores como Charles Ford (1999), Robert Trivers (2000) y David Livingstone Smith (2005), quienes privilegian la hipótesis de que el autoengaño surgió primeramente como una estrategia para mentir de forma más convincente.

\section{Referencias}

Adenzato, M. y Ardito, R. 1999. The role of theory of mind and deontic reasoning in the evolution of deception. En Hahn, M. y Stoness, S. (Eds.). Proceedings of the Twenty-First Conference of the Cognitive Science Society (pp. 7-12). Mahwah, New Jersey: Lawrence Erlbaum Associates.

Arce, M. 2002. Visitas al desván. San José: Editores Alambique.

2004. De leguas y minutos. San José: Editores Alambique.

2005. Las huellas del zapatero. San José: Editores Alambique.

.2008. Análisis de tropa y análisis literario: Una aproximación al Nibelungenlied. Kañina, 32 (2), 109-122.

2010. Marco para una teoría de la representación en ciencia cognitiva. Humanitas, 7 (7), 58-72.

2011. Algunos principios de la Teoría de Dinámica de Tropas. Humanitas, 8 (8), 71-97. 
Byrne, R. 2003. Tracing the evolutionary path of cognition. En Brune, M., Ribbert, H. y Schiefenhovel, W. (Eds.). The social brain: Evolution and pathology (pp. 43-60). Chichester, West Sussex: John Wiley \& Sons.

Byrne, R. y Bates, L. 2007a. Brain evolution: When is a group not a group? Current Biology, 17 (20), R883-R884.

. 2007). Sociality, evolution and cognition review. Current Biology, 17 (16), R714-R723.

Byrne, R. y Whiten, A. 1990. Tactical deception in primates: The 1990 database. Primate Report, 27, 1-101.

. 1992. Cognitive evolution in primates: Evidence from tactical deception. Man, 27, 609-627.

Cartín, J. 2009. Emoción, sociabilidad y lenguaje: Propuesta de un modelo cognoscitivo de las emociones en su dimensión social. San José: EUNED.

D’Alton, C. 2006. El origen del lenguaje. Tesis elaborada como requisito de graduación en la Maestría en Ciencias Cognoscitivas, Universidad de Costa Rica.

De Waal, F. (Ed.). 2001. Tree of origin: What primate behavior can tell us about human social evolution. Cambridge, Massachusetts: Harvard University Press.

De Waal, F. 2005. Our inner ape: The best and the worst of human nature. London: Granta Books.

Deacon, T. 1997. The Symbolic Species. New York: W.W. Norton \& Company.
DePaulo, B., Kashy, D., Kirkendol, S., Wyer, M. y Epstein, J. 1996. Lying in everyday life. Journal of Personality and Social Psychology, 70 (5), 979-995.

Dunbar, R. y Shultz, S. 2007a. Evolution in the social brain. Science, 317, 1344-1347

. 2007b. Understanding primate brain evolution. Phil. Trans. R. Soc. B, 362, 649-658.

Feldman, S., Forrest, J. y Happ, B. 2002. Selfpresentation and verbal deception: Do Self-presenters lie more? Basic and Applied Social Psychology, 24 (2), 163-170.

Ford, C. 1999. Lies! Lies!! Lies!!!: The psychology of deceit. Washington, D.C.: American Psychiatric Press.

Hauser, M. 1997. Minding the behaviour of deception. En Whiten, A. y Byrne, R. (Eds.). Machiavellian intelligence II: Extensions and evaluations (pp. 112-143). Cambridge: Cambridge University Press.

McFarland, D. y Bösser, T. 1993. Intelligent behavior in animals and robots. Cambridge, Massachusetts: The MIT Press.

Mitchell, R. 1993. Animals as liars: The human face of nonhuman duplicity. En Lewis, $M$. y Saarni, C. (Eds.). Lying and deception in everyday life (pp. 59-89). New York: The Guildford Press.

Mitchell, R. y Thompson, N. (Eds.). 1986. Deception: Perspectives on human and nonhuman deceit. New York: State University of New York Press.

Shultz, S. y Dunbar, R. 2007. The Evolution of the Social Brain: Anthropoid Primates 
Contrast with Other Vertebrates. Proc. $R$. Soc. B, 274, 2429-2436.

Smith, D. (2005, 18 de mayo). Natural-born liars: Why do we lie, and why are we so good at it? Because it works. Scientific American.

Stevens, M. y Merilaita, S. 2009. Animal camouflage: Current issues and new perspectives. Phil. Trans. R. Soc. B, 364, 423-427.

Trivers, R. 2000. The elements of a scientific theory of self-deception. En LeCroy, D. y Moller, P. (Eds.). Annals of the New York Academy of Sciences: Evolutionary perspectives on human reproductive behavior (Vol. 907, pp. 114-131). New York: New York Academy of Sciences.

Whiten, A. y van Schaik, C. 2007. The evolution of animal 'cultures' and social intelligence. Phil. Trans. R. Soc. B, 362, 603-620.

Wilson, R. y Keil, F. (Eds.). 1999. The MIT Encyclopedia of the Cognitive Sciences. Cambridge, Massachusetts: The MIT Press. 